\title{
Profile and management of patients at a memory clinic
}

\author{
Sebastiana Zimba Kalula, Monica Ferreira, Kevin G F Thomas, Linda de Villiers, John A Joska, Leon N Geffen
}

Increasing longevity and a growing older population are being accompanied by a higher prevalence of dementia and concomitant demand for care. In this connection, the University of Cape Town/Groote Schuur Hospital (UCT/ GSH) Memory Clinic provides a valuable service to patients, families and health professionals. High levels of behavioural and psychological symptoms of dementia need expert tertiary level assessment and management. Public education on dementia, early referral for assessment by primary care health professionals, and advanced training of health professionals are needed to encourage early recognition and appropriate management. Community-based care services too are needed to support caregivers of cognitively impaired older individuals.

S Afr Med J 2010; 100: 449-451.
Memory clinics were first introduced in the USA in the mid-1970s and have since become widely established in the developed world. ${ }^{1}$ Their initial purpose was mainly research but this changed when treatment became available for Alzheimer's disease (AD). A main aim at present is to provide, and improve, high-quality dementia care. Memory clinics facilitate early diagnosis of dementia, disseminate information, initiate and monitor treatment, educate clients and their families, and provide training and research opportunities for medical and mental health professionals. ${ }^{1}$ Few memory clinics have been established in sub-Saharan Africa (SSA); of those few, most are in South Africa.

The prevalence of dementia in SSA and South Africa has not been established conclusively. ${ }^{2}$ Studies have reported consistently lower rates of dementia than in developed countries, which has been attributed to differential survival rates; non-presentation of cases of mental illness at service facilities owing to stigma and other reasons; access barriers to health care; symptoms and signs of dementia being accepted by sufferers' families as normal in ageing; and preferential use of traditional healing or complementary medicine. ${ }^{3}$

Despite the overall lower life expectancy in SSA, individuals aged 60 and over are projected to increase from 35 million in 2006 , to 139 million by 2050 - an increase from $5 \%$ to $8 \%$ of the population. ${ }^{4}$ Over the same period, South Africa's older population is projected to increase from 3.3 million to 6.4 million - an increase from $7 \%$ to $13 \%$ of the country's

Division of Geriatric Medicine, Institute of Ageing in Africa, Department of Medicine, Faculty of Health Sciences, University of Cape Town

Sebastiana Zimba Kalula, MB ChB, MMed, MPhil, FRCP (UK)

Linda de Villiers, MB ChB, FCP (SA)

Leon N Geffen, MB ChB, FCFP (SA)

International Longevity Centre - South Africa, Faculty of Health Sciences, University of Cape Town

Monica Ferreira, DPhil

ACSENT Laboratory, Department of Psychology, University of Cape Town Kevin G F Thomas, PhD

Division of Neuropsychiatry, Department of Psychiatry and Mental Health, University of Cape Town

John A Joska, MB ChB, MMed (Psych), FCPsych (SA) population. A greater number of older people will be at risk of age-related disease, including the dementias, that will translate into increased demand for their management.

\section{The UCT/GSH Memory Clinic}

The University of Cape Town/Groote Schuur Hospital (UCT/GSH) Memory Clinic was established in 1999 in response to increasing numbers of patients presenting with a memory disorder in the geriatrics clinics, and for whom multidisciplinary management was poorly co-ordinated. The weekly half-day clinic is run by two geriatricians, a psychogeriatrician and a neuropsychologist, with access to a hospital nurse, social worker and occupational therapist as needed. Referrals are accepted from general practitioners, community health centres and other specialists. Patients undergo initial neuropsychological screening, physical examination and guided special investigations. They are followed up and stabilised before returning to the community. A caregiver accompanies a patient to the clinic and is interviewed at each visit regarding the patient's symptoms and care.

We studied all patients $(N=305)$ seen at the clinic between 2003 and 2008 to evaluate the role and function of the clinic in a resource-limited context. The findings provided a patient profile, and an indication of diagnostic and management trends and the challenges of operating the clinic.

Permission for the study was obtained from the Human Research Ethics Committee of the UCT Faculty of Health Sciences. Socio-demographic variables, cognitive history, behavioural/personality changes, and medical and functional history for each patient were gathered, using a standard assessment protocol. Diagnoses were made according to standard criteria, ${ }^{15-17}$ and most were conducted by a consensus of the team members that included a management plan.

A standardised assessment protocol to assess patients comprised:

- a neuropsychological assessment battery comprising the Mini-Mental State Examination (MMSE) ${ }_{,}^{5}$ verbal fluency tests, ${ }^{6}$ digit span test, ${ }^{7}$ Rey Auditory Verbal Learning Test, ${ }^{8}$ scene drawing test, CLOX executive clock drawing test, ${ }^{9}$ Luria hand sequence and recursive figures tests, ${ }^{10}$ and the Trail Making Test ${ }^{11}$ 
- the modified Bristol Activities of Daily Living scale $\left(\right.$ BADLS) ${ }^{12}$

- the Cornell Scale for Depression ${ }^{13}$

- the Neuropsychiatric Inventory and Caregiver Distress (NPI-D) Scale ${ }^{14}$ to screen behavioural and psychological symptoms.

\section{Patient profile}

\section{Socio-demographic characteristics}

The patients' mean age was 70 years $(\mathrm{SD} \pm 10.26)$ (range 37 89 years); $61 \%$ were female, $49 \%$ were married and $34 \%$ were widowed. The ethnic distribution was $64.9 \%$ coloured, $28.5 \%$ white, $6.6 \%$ black, and $2 \%$ Indian, which differs from that of older persons in the community that the clinic serves ( $44 \%$ coloured, $44 \%$ white, $11 \%$ black, and $0.9 \%$ Indian $)^{18}$ and distributions of older patients in other hospital departments (13\% of elderly patients who access the Emergency Department are black). Stroke predisposes to the development of dementia, and is more common in blacks than in whites. ${ }^{19}$ Therefore it would be expected that more black patients would be at risk of, and present with a memory disorder consequent to, the development of vascular dementia. The proportion of white patients in the clinic (26\%) is similarly lower than the $44 \%$ proportion of older whites in the hospital's catchment area population. Older whites who are more affluent may have access to private health care, whereas the poor rely on public health care.

Family members cared for $79 \%$ of the patients, $6 \%$ were institutionalised, $10 \%$ lived alone, and $74 \%$ of those who lived with family lived with a spouse and/or adult children. Scant or no formal support is available to carers of such patients in South Africa. Few residential or respite care facilities are available, and numerous individuals with dementia must be cared for at home by family members. Primary caregivers consequently carry a heavy social and financial burden; some cease formal employment because of caregiving responsibilities. A cognitively impaired older individual's inability to perform daily activities, and the presence of behavioural and psychological symptoms, contribute to caregiver stress. ${ }^{20}$ However, the comparatively low number of institutionalised patients could be due to cultural preferences, economic factors and availability of care from family members. ${ }^{21}$ Older individuals with a memory disorder who live alone are vulnerable and constitute a high-risk group as there are few community support services, and access to oldage homes is limited.

\section{Clinical diagnoses}

Patients diagnosed with dementia comprised $74 \%$, and those with mild cognitive impairment (MCI) $8 \% ; 15 \%$ (46) were diagnosed with depression, and 3\% (10) with depression and dementia; 8 had other medical diagnoses but no cognitive impairment, and 5 had other psychiatric diagnoses. Of the patients with dementia, $44 \%$ had $\mathrm{AD}, 28 \%$ had vascular dementia, 15\% had mixed Alzheimer's and vascular dementia, and 13\% (36) had other forms of dementia, namely Lewy body dementia (8), Parkinson's disease with dementia (2), frontotemporal dementia (2), HIV-associated dementia (1), alcoholrelated dementia (10), history of previous head injury (4) and undetermined (3).

Hence, dementia was the most common diagnosis and $\mathrm{AD}$ the predominant subtype. Unlike most other types of dementia, AD is characterised by primary memory deficit and progressive memory loss over the course of the disease. While memory clinics in developed countries have reported prevalence rates of $50-62 \%$ for AD and $7-20 \%$ for vascular dementia $(\mathrm{VaD}){ }^{22}$ our audit showed an AD prevalence of $44 \%$, compared with $36 \%$ in a Brazilian memory clinic. ${ }^{23}$ Our high prevalence of $\mathrm{VaD}(28 \%)$ and mixed dementia (15\%) suggests a high prevalence of stroke and attendant risk factors that may be inadequately managed.

Cognitive decline of $\geq 3$ years' duration was reported in $44 \%$ of patients (range $<1-10$ years). Memory loss was the first symptom noticed by a caregiver in $90 \%$ of cases. Behavioural and psychological symptoms of dementia (BPSD) were the most common precipitants, with caregivers commonly reporting anxiety $(28 \%)$, apathy $(28 \%)$, pacing $(24 \%)$, hallucinations $(19 \%)$, delusions $(19 \%)$ and wandering $(16 \%)$. In most cases, BPSD had been present for $>2$ years. Personality change was reported in $61 \%$ of patients - most commonly, increased irritability (45\%) and increased aggression (34\%).

Patients typically presented at an advanced stage of worrisome behavioural and psychological symptoms. The presence of BPSD increased caregiver burden, and was corroborated by a high correlation between behavioural disturbance (NPI) and NPI-Distress scores $(r=0.85, p<0.001)$, and a correlation between impaired activities of daily living (ADL) function and a NPI-Distress score $(r=0.27, p=0.004)$. Caregivers commonly showed signs of distress at presentation and of needing support and counselling.

\section{Patient management}

Optimal management of patients with dementia requires nonpharmacological and pharmacological regimens to control BPSD and maintain functional independence.

\section{Drug management}

Although depression was diagnosed in $15 \%$ of the patients, antidepressants were prescribed for more than $43 \%$, sometimes for their sedative properties or management of apathy, rather than for depression per se.

The most commonly prescribed drugs to manage BPSD were traditional antipsychotic agents: haloperidol, thioridazine (prior to its discontinuation by the manufacturers) and chlorpromazine. Atypical antipsychotic agents are restricted in public hospitals owing to their high cost. The acetylcholinesterase inhibitors are known to ameliorate BPSD and maintain functioning without influencing disease progression. ${ }^{24}$ The drugs are freely available in the private sector, and their benefits are discussed with patients, but only $7(2 \%)$ who could afford them had them prescribed. 
Consequently, most of the clinic's patients are excluded from appropriate pharmacological treatment options because of resource constraints.

\section{Benefits of a memory clinic}

Patients and their caregivers benefit from management at the memory clinic. Patients receive a full physical assessment and management of cognitive functioning. Patients and their caregivers receive counselling and support. Follow-up provides optimal care for co-morbid conditions such as vascular risk factors which may accelerate the progression of the cognitive disorder. Carers benefit from education that gives them insight into memory, behaviour and personality changes that may occur as the disorder progresses. Carers are empowered to manage the patient at home and to plan for the future. Counselling and support enhance their coping abilities, and reduce caregiving stress. Carers are referred to community resources where available, such as dementia support groups and respite care services, and encouraged to manage their own health optimally.

The clinic benefits health professionals and students trained at the site and provides for hospital- and communitybased studies on memory disorders (including multi-centre pharmaceutical research). The clinic has links with agencies that advocate for public education, support and awareness of memory disorders as a disease entity that needs timely and appropriate identification and management. Overall, the clinic is valuable in a continuum of health care services for older persons.

\section{Challenges for the memory clinic}

Three major challenges are experienced in clinical diagnoses at the memory clinic. Firstly, the MMSE and other tests in the neuropsychology battery used to screen for dementia may be inappropriate because of the low education level of many patients, and their non-validation in this population. Coloured and black patients generally have a lower education level than white patients: $\leq 7$ years' education in $45 \%$ of coloured and black patients, compared with $5 \%$ in white patients. Low education leads to a false positive screening for dementia. A minimum of Grade 8 reading skill is required for effective employment of the MMSE. ${ }^{25}$ Consequently, the diagnosis of dementia in our patients with low education levels had to be largely based on the caregivers' reports of impaired ADLs and/ or instrumental activities of daily living (IADLs) in the absence of a physical disability. A study to validate locally adapted instruments is in progress.
Secondly, although all diagnoses are based on defined criteria, patients do not undergo routine supportive brain imaging, which may influence diagnostic accuracy. Thirdly, a relative lack of awareness of the possibility of a patient having a memory disorder on the part of health professionals at a primary care level and family members diminishes an opportunity for referral for early diagnosis and prompt management of the disorder.

References

1. Jolley D, Benbow SM, Grizzell M. Memory clinics. Postgrad Med J 2006; 82: 119-206.

2. Kalaria RN, Maestre GE, Arizaga R, et al. Alzheimer's disease and vascular dementia in developing countries: prevalence, management, and risk factors. Lancet Neurol 2008; $7(9)$ $812-826$.

3. Ineichen B. The epidemiology of dementia in Africa: a review. Soc Sci Med 2000; 50: 1673-1677.

4. United Nations Population Division. World Population Prospects, 2004. UNPD: New York, 2006

5. Folstein MF, Folstein SE, McHugh PR. Mini-Mental State: a practical method for grading the cognitive state of patients for the clinician. J Psych Res 1975; 12: 189-198.

6. Strauss E, Sherman EMS, Spreen O. A Compendium of Neuropsychological Tests: Administration, Norms, and Commentary. 3rd ed. New York: Oxford University Press, 2006.

7. Lezak MD, Howieson DB, Loring DW. Neuropsychological assessment. 4th ed. New York: Oxford University Press, 2004.

8. Rey A. L'examen Clinique en Psychologie. Paris: Presse Universitaires de France, 1958.

9. Royall DR, Cordes JA, Polk M. Clox: an executive clock drawing task. J Neurol Neurosurg Psychiatry 1998; 64: 588-594.

10. Luria AR. Higher Cortical Functions in Man (B. Haigh, Trans.). London: Tavistock, 1966 (original work published 1962).

11. Reitan RM. The relation of the Trail Making Test to organic brain damage. Journal of Consulting Psychology 1955; 19: 393-394.

12. Bucks RS, Ashworth DL, Wilcock GK, Siegfried K. Assessment of activities of daily living in dementia: Development of the Bristol activities of daily living scale. Age Ageing 1996; 25 $113-120$.

13. Alexopoulos GS, Abrams RC, Young RC, Shamoian CA. Cornell scale for depression in dementia. Biol Psychiatry 1988; 23: 271-284.

14. Kaufer DI, Cummings JL, Christine D, et al. Assessing the impact of neuropsychiatric symptoms in Alzheimer's disease: the Neuropsychiatric Inventory Caregiver Distress Scale. J Am Geriatr Soc 1998; 46: 210-215.

15. American Psychiatric Association. Diagnostic and Statistical Manual of Mental Disorders. 4th ed. Washington, DC: American Psychiatric Press, 1994.

16. McKhann G, Drachman D, Folstein M, et al. Clinical diagnosis of Alzheimer's disease: Report of the NINCDS-ADRDA work group under the auspices of the Department of Health and Human Services Task force on Alzheimer's disease. Neurology 1984; 34: 939-944.

17. Roman GC, Tatemichi TK, Erkinjuntti T, et al. Vascular dementia: Diagnostic criteria for research studies. Report of the NINDS-AIREN International Workshop. Neurology 1993; 43 250-260.

18. Statistics South Africa. Mid-Year Population Estimates, 2008. Pretoria: Statistics SA, 2008. www. statssa.gov.za/publications/P0302/P03022008.pdf (accessed 27 October 2008).

19. Opie LH, Seedat YK. Hypertension in sub-Saharan African populations. Circulation 2005; 112 3562-3568.

20. Tan LL, Wong HB, Allen $\mathrm{H}$. The impact of neuropsychiatric symptoms of dementia on distress in family and professional caregivers in Singapore. Int Psychogeriatr 2005; 17(2): 253263.

21. Amoateng AY, Heaton TB, Kalula-Sabiti J. Living arrangements in South Africa. In: Amoateng AY, Heaton TB, eds. Families and Households in Post-Apartheid South Africa. Cape Town: HSRC, 2007

22. Clarfield AM. The decreasing prevalence of reversible dementias. An updated meta-analysis. Arch Intern Med 2003; 163: 2219-2229.

23. Vale FAC, Miranda SJC. Clinical and demographic features of patients with dementia attended in a tertiary outpatient clinic. Arg Neuropsiquiatr 2002; 60(3-A): 548-552.

24. Holden M, Kelly C. Use of cholinesterase inhibitors in dementia. Advances in Psychiatric Treatment 2002; 8: 89-96

25. Weiss BD, Reed R, Kligman EW, Abyad A. Literacy and performance on the Mini-Mental State Examination. J Am Geriatr Soc 1995; 43(7): 807-810.

Accepted 25 November 2009. 